\title{
Oxymatrine Inhibits Proliferation and Migration of Vulvar Squamous Cell Carcinoma Cells via Attenuation of the RAS/RAF/MEK/ERK Pathway
}

This article was published in the following Dove Press journal: Cancer Management and Research

\section{Yanshi Wang (D) \\ Shaojie Yang (D) \\ Shanshan Zhang (DD \\ Xin Wu}

Department of Gynecology, The First Affiliated Hospital of China Medical University, Shenyang, Liaoning II000I, People's Republic of China
Correspondence: Xin Wu

The First Affiliated Hospital of China Medical University, 155 Nanjing North

Road, Shenyang, Liaoning I 10000 ,

People's Republic of China

Tel +86-|3940408|8|

Fax+8624-83696110

Email wuxin@cmu.edu.cn
Purpose: To evaluate the anti-tumor effects of oxymatrine in vulvar squamous cell carcinoma (VSCC) cells and to explore the underlying mechanisms.

Methods: We selected SW962 and A431 VSCC cell lines. Cell proliferation was examined using MTT assay. Cell cycle and apoptosis were detected using flow cytometry. Migration and invasion were evaluated using transwell and wound-healing assays. The relevant protein expression and signaling pathways were analyzed using Western blotting.

Results: Oxymatrine inhibited the proliferation of SW962 and A431 VSCC cells in a timeand dose-dependent manner. Oxymatrine induced cell cycle arrest in the G2/M phase by increasing the protein expression of P21 and decreasing levels of cyclin B1 and CDC2. Oxymatrine upregulated the expression of cleaved-caspase 3 and BAX and downregulated the expression of BCL2, which led to an increase in apoptosis. Oxymatrine also suppressed the migration and invasion of SW962 and A431 cells by reducing levels of MMP2 and MMP9. After treatment with oxymatrine or a RAS inhibitor (salirasib), expression levels of RAS, p-RAF, p-MEK, p-ERK, C-MYC, and MMP2 were reduced. When TGF- $\beta 1$ was used to stimulate SW962 and A431 cells, the expression of the above proteins increased; this increase was reversed by using oxymatrine or salirasib again.

Conclusion: Oxymatrine inhibits proliferation and migration of VSCC cells by blocking the RAS/RAF/MEK/ERK pathway.

Keywords: oxymatrine, vulvar squamous cell carcinoma, RAS/RAF/MEK/ERK

\section{Introduction}

Vulvar squamous cell carcinoma (VSCC) accounts for about 90\% vulvar malignant tumors. The main clinical treatment is surgery, supplemented by radiotherapy and chemotherapy. The 5-year survival rate during the early stage is $80-90 \%$, decreasing to $25-50 \%$ in the late stage. ${ }^{1,2}$ The leading causes of death and poor clinical outcomes for patients with VSCC are recurrence and metastasis. The limitations of local radiotherapy of the vulva and the resistance of the tumor to radiotherapy and chemotherapy mean that current treatment methods cannot suppress the growth of tumors. This has prompted clinicians to search for better therapeutic strategies. In cancer research, traditional Chinese medicine has attracted much attention because of its multiple targets and small side effects. Oxymatrine is a quinazine alkaloid extracted from Sophora flavescens. Its molecular formula is $\mathrm{C}_{5} \mathrm{H}_{24} \mathrm{~N}_{2} \mathrm{O}_{2}$, and it is a hydrophilic monomer. ${ }^{3}$ Some research has shown that oxymatrine has anti-tumor effects in breast cancer, non-small-cell lung cancer, cervical cancer, and bladder 
cancer cells. $^{4-7}$ Oxymatrine can inhibit the proliferation and migration of tumor cells by inhibiting the p38, EGFR/ $\mathrm{PI} 3 \mathrm{~K} / \mathrm{AKT} / \mathrm{mTOR}$, and MEK-1/ERK1/2/MMP2 signaling pathways. $^{8-10}$

RAS is a proto-oncogene with a mutation rate of over $30 \%$ in human tumors, of which KRAS accounts for $20 \%$, NRAS for $8 \%$, and HRAS for $3.3 \%$ of mutations. ${ }^{11}$ Abnormal mutations of Ras have been reported to be detected in approximately $10.2 \%$ of vulvar squamous cell carcinomas and are associated with poor prognosis. ${ }^{12}$ RAS activates a variety of signaling pathways, including RAF/ MEK/ERK, PI3K/AKT, and RalGEF/Ral, which are involved in cell proliferation, invasion, and apoptosis. $^{11,13}$ The RAF/MEK/ERK signaling pathway is currently considered to be the most prominent and classical of these pathways. Abnormal mutations of RAS and BRAF can be detected in VSCC, ${ }^{14}$ which are transmitted to proteins through transcription or translation, leading to sustained phosphorylation and activation of ERK. ${ }^{15}$ Abnormal activation of the KIT/NRAS/BRAF/ERK signaling pathway has been reported in clinical specimens of vulvar melanoma. ${ }^{16}$ Therefore, we speculate that the abnormal activation of the RAS/RAF/MEK/ERK signaling pathway may promote the proliferation and invasion of VSCC cells, and oxymatrine can inhibit the above process.

\section{Materials and Methods}

\section{Cell Culture and Treatment}

The SW962 cell line, derived from a lymph node metastasis of vulvar carcinoma, was bought from the ATCC cell bank (Manassas, VA, USA). The A431 cell line, established from solid tumors, was obtained from Professor Lu Chunyang (Cancer Hospital of the University of Chinese Academy of Sciences). Cells were maintained in Dulbecco's modified Eagle's medium (DMEM) with 10\% FBS (Biological Industries, Kibbutz Beit-Haemek, Israel) at $37{ }^{\circ} \mathrm{C}$ in a sterile $5 \% \mathrm{CO}_{2}$ environment. Subsequent experiments were carried out using cells in the logarithmic growth phase.

\section{Chemicals and Reagents}

Oxymatrine (purity $>$ 98\%, Aladdin Technology Co., Ltd., Shanghai, China) was dissolved in DMEM to prepare a stock solution at a concentration of $10 \mathrm{mg} / \mathrm{mL}$ and stored at $4{ }^{\circ} \mathrm{C}$. TGF- $\beta 1$ was purchased from Solarbio Biotechnology Co., Ltd. (Beijing, China). Salirasib was purchased from Selleck Chemicals (Houston, TX, USA). MTT was obtained from
Invigentech (Xi'an, China). The transwell apparatus and Matrigel were bought from BD Biosciences (Franklin Lakes, NJ, USA). The cell cycle and apoptosis assay kits were purchased from Nanjing KeyGen Biotechnology Co., Ltd. (Nanjing, China). Primary antibodies: anti-RAS (ab52939; 1:30,000), anti-RAF (ab200653; 1:1000), antiphospho(p)-RAF (ab173539; 1:5000), anti-P21 (ab109520; 1:5000), anti-cyclin B1 (ab32053; 1:50,000), anti-CDC2 (ab39688; 1:500), anti-cleaved-caspase 3 (ab32042; 1:500), anti-BCL2 associated X protein (BAX; ab32503; 1:5000), anti-B-cell CLL/lymphoma 2 (BCL2; ab32124; 1:500), antimatrix metalloproteinase (MMP)-2 (ab97779; 1:2000), antiMMP-9 (ab38898; 1:500), anti-glyceraldehyde-3-phosphate dehydrogenase (GAPDH; ab9485; 1:2500) and anti-C-MYC (ab39688; 1:500) were bought from Abcam (Cambridge, MA, USA). Anti-MEK (ab200653; 1:1000), anti-p-MEK (\#9121; 1:1000), anti-ERK (\#9102; 1:1000), and antip-ERK (\#9101; 1:1000) were acquired from Cell Signaling Technology (Danvers, MA, USA). The secondary antibody, horseradish peroxidase-conjugated goat anti-rabbit-IgG (ab205718; 1:50,000), was acquired from Abcam (Cambridge, MA, USA).

\section{MTT Assay}

Cells were seeded in 96-well plates at $5 \times 10^{3}$ cells/well and incubated for $24 \mathrm{~h}$. Oxymatrine with a final concentration of $0.25 \mathrm{mg} / \mathrm{mL}, 0.5 \mathrm{mg} / \mathrm{mL}, 1 \mathrm{mg} / \mathrm{mL}, 2 \mathrm{mg} / \mathrm{mL}, 4 \mathrm{mg} / \mathrm{mL}$, or $6 \mathrm{mg} / \mathrm{mL}$ was used for the intervention, and cells were cultured for $24 \mathrm{~h}, 48 \mathrm{~h}$, and $72 \mathrm{~h}$, respectively. Then, $20 \mu \mathrm{L}$ MTT solution was added in the dark. After $4 \mathrm{~h}$ incubation, we immediately replaced the medium with $150 \mu \mathrm{L}$ of DMSO. An enzyme microplate reader was used to measure the absorbance at $490 \mathrm{~nm}$ (Thermo Scientific, Waltham, MA, USA).

\section{Cell Cycle Assay}

Cells were cultured with $1.5 \mathrm{mg} / \mathrm{mL}$ or $3 \mathrm{mg} / \mathrm{mL}$ oxymatrine for $48 \mathrm{~h}$ and harvested. After fixing with $70 \%$ ice ethanol at $4{ }^{\circ} \mathrm{C}$ overnight, $500 \mu \mathrm{L}$ of propidium iodide/ RNase A staining solution, prepared in advance, was added to the samples to stain them for $30 \mathrm{mins}$ in the dark. Data were acquired and analyzed using Mod-Fit version 4.1 (BD Biosciences, San Jose, CA, USA).

\section{Apoptosis Assay}

Cells were cultured with $1.5 \mathrm{mg} / \mathrm{mL}$ or $3 \mathrm{mg} / \mathrm{mL}$ oxymatrine for $48 \mathrm{~h}$ and harvested. After washing and resuspending the cells, staining reagents were added to the samples 
and reacted for $15 \mathrm{~min}$ in the dark. Data were acquired and measured using FACSDiva version 6.2 (BD Biosciences, San Jose, CA, USA).

\section{Transwell Assay}

After resuspension in serum-free DMEM, a $150-\mu \mathrm{L}$ cell suspension with a density of $1 \times 10^{5}$ cells/well was placed in the upper chamber of the transwell apparatus, and $600 \mu \mathrm{L}$ of DMEM medium containing 10\% FBS was added to the bottom chamber. Cells continued to culture for $48 \mathrm{~h}$. The upper surface of the chamber was wiped with a wet cotton swab and fixed with 4\% paraformaldehyde for $30 \mathrm{~min}$, and with $0.1 \%$ crystal violet for $30 \mathrm{~min}$. Finally, 10 fields of view were randomly selected under a microscope for imaging and counting. For the invasion experiment, Matrigel was diluted to a $0.5 \mathrm{mg} / \mathrm{mL}$ working concentration in serum-free DMEM. Then, a $10 \mu \mathrm{L}$ Matrigel dilution was coated onto each upper apparatus and solidified for $4 \mathrm{~h}$. Fixing, staining, and counting were then carried out according to the protocol described above.

\section{Wound Healing Assay}

After seeding an equal number of cells in a six-well plate, a $200 \mu \mathrm{L}$ sterile tip was used to scratch the cells, creating a straight line. The exfoliated cell mass was washed off with phosphate-buffered saline until the scratch area was clean. Serum-free oxymatrine medium was added and incubated for $48 \mathrm{~h}$. The scratch width was measured at $0 \mathrm{~h}$ and 48 $\mathrm{h}$ under an inverted microscope (Nikon, Tokyo, Japan).

\section{Western Blotting Assay}

The collected cells were lysed on ice for 20 min using precooled radioimmunoprecipitation assay lysis buffer and centrifuged at $16,097 \times \mathrm{g}$ for $20 \mathrm{~min}$ at $4{ }^{\circ} \mathrm{C}$. After sodium dodecyl sulfate polyacrylamide gel electrophoresis of identical amounts $(40 \mu \mathrm{g})$ of protein, the target proteins were electro-transferred to a polyvinylidene fluoride membrane. The membrane was blocked with $5 \%$ skimmed milk for $2 \mathrm{~h}$, and incubated with primary antibodies overnight at $4{ }^{\circ} \mathrm{C}$ and secondary antibodies for $2 \mathrm{~h}$ at room temperature. The immunoreactive proteins were detected using intensive chemiluminescence. The ImageJ software was used to measure the gray values of bands.

\section{Statistical Analysis}

Data analysis was performed by GraphPad Prism 6.0 and the data were expressed as mean \pm standard error. The Student's $t$-test was used to evaluate the differences between the control group and the experimental group, and one-way analysis of variance followed by Tukey's test was used to evaluate the differences among multiple groups. $\mathrm{P}<0.05$ was considered statistically significant. All experiments were repeated three times.

\section{Results}

\section{Oxymatrine Inhibits the Proliferation of SW962 and A43I Cells}

The MTT results revealed that oxymatrine reduced the proliferation of SW962 and A431 cells in a time- and dose-dependent manner. The half maximal inhibitory concentration $\left(\mathrm{IC}_{50}\right)$ values of oxymatrine for SW962 cells were $4.369 \mathrm{mg} / \mathrm{mL}, 3.229 \mathrm{mg} / \mathrm{mL}$, and $2.350 \mathrm{mg} / \mathrm{mL}$ at $24 \mathrm{~h}, 48 \mathrm{~h}$, and $72 \mathrm{~h}$, respectively ( $<<0.05$; Figure 1A). The $\mathrm{IC}_{50}$ values of oxymatrine for A431 cells were $4.342 \mathrm{mg} / \mathrm{mL}, 3.524 \mathrm{mg} / \mathrm{mL}$, and $2.425 \mathrm{mg} / \mathrm{mL}$ at $24 \mathrm{~h}$, $48 \mathrm{~h}$, and $72 \mathrm{~h}$, respectively $(\mathrm{P}<0.05$; Figure $1 \mathrm{~B})$. Correspondingly, we used 1.5 and $3 \mathrm{mg} / \mathrm{mL}$ oxymatrine for the subsequent experiments.

\section{Oxymatrine Induces Cell Cycle Arrest in G2/M Phase in SW962 and A43I Cells}

The effects of oxymatrine on the cell cycle of VSCC cells was assessed using flow cytometry assays. After treatment with oxymatrine at 1.5 and $3 \mathrm{mg} / \mathrm{mL}$ for $48 \mathrm{~h}$, the proportion of cells in the $\mathrm{G} 2 / \mathrm{M}$ phase from $4.2 \pm 0.5 \%$ increased to $14.3 \pm 2.3 \%$ and $22.8 \pm 1.1 \%$ in SW962 cells, and from $4.4 \pm 1.2 \%$ increased to $13.8 \pm 1.7 \%$ and $21.5 \pm 2.2 \%$ in $\mathrm{A} 431$ cells, respectively $(\mathrm{P}<0.05$; Figure $2 \mathrm{~A}-\mathrm{C})$. The Western blot assay revealed that oxymatrine downregulated cyclin B1 and CDC2, whereas it enhanced the expression of P21, in both SW962 and A431 cells ( $<<0.05$; Figure 2D-G). These results indicated that oxymatrine induced cell cycle arrest in G2/M phase in SW962 and A431 cells.

\section{Oxymatrine Promotes Apoptosis of SW962 and A43I Cells}

We further evaluated the effects of oxymatrine on apoptosis of VSCC cells. As shown in Figure $3 \mathrm{~A}-\mathrm{C}$, after treatment with oxymatrine at 1.5 and $3 \mathrm{mg} / \mathrm{mL}$ for $48 \mathrm{~h}$, the proportion of apoptotic cells from $11.6 \pm 0.7 \%$ increased to $21.6 \pm 1.2 \%$ and $41.1 \pm 1.0 \%$ in SW962 cells, and from 14.1 $\pm 1.4 \%$ increased to $17.8 \pm 0.2 \%$ and $35.8 \pm 0.7 \%$ in $\mathrm{A} 431$ cells, respectively $(\mathrm{P}<0.05)$. As shown in Figure $3 \mathrm{D}-\mathrm{G}$, oxymatrine significantly increased levels of cleavedcaspase 3 and BAX and decreased BCL2 expression in 

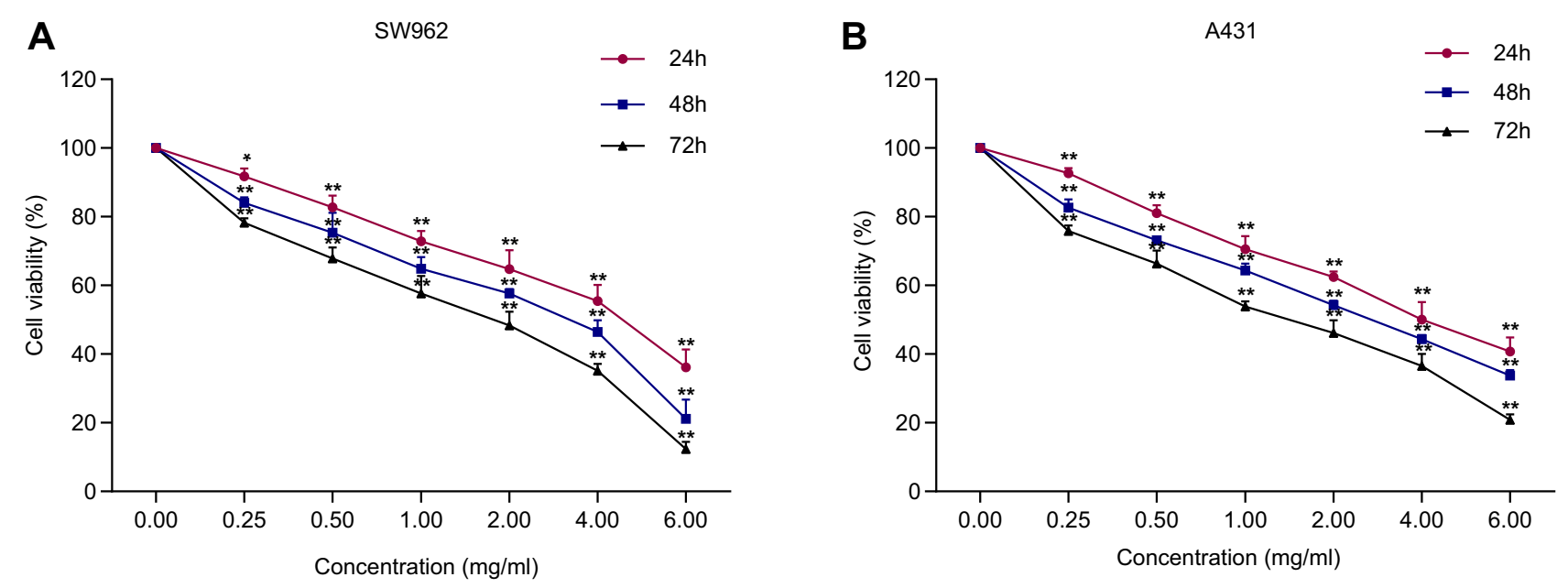

Figure I Oxymatrine inhibits the viability of SW962 and A43I cells. (A-B) After treatment with oxymatrine for $24 \mathrm{~h}, 48 \mathrm{~h}$, and $72 \mathrm{~h}$, the viability of SW962 and A43 I was measured. $* \mathrm{P}<0.05, * * \mathrm{P}<0.01$ compared with $0 \mathrm{mg} / \mathrm{mL}$ oxymatrine.

A

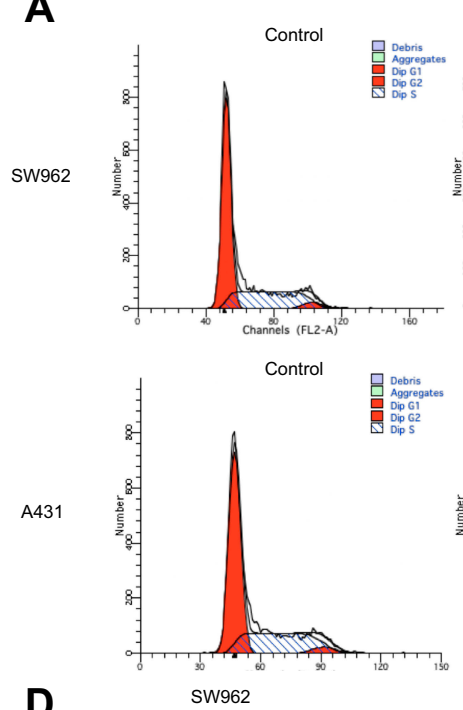

D

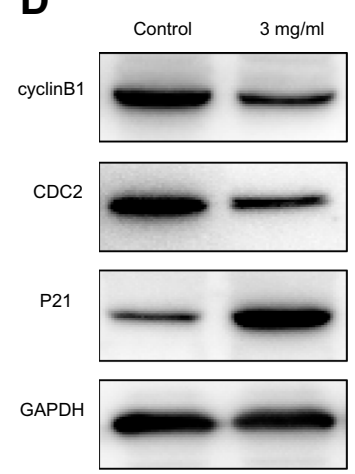

\section{E}

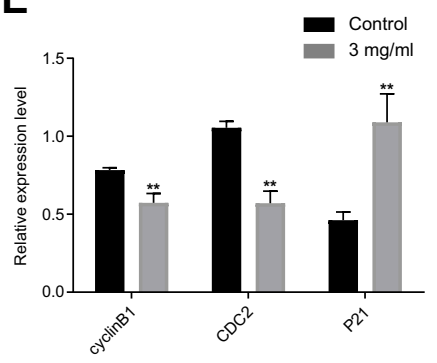

B

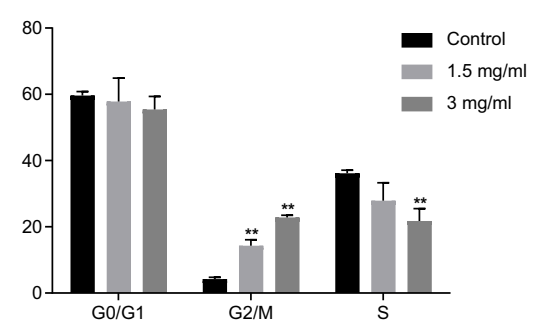

C

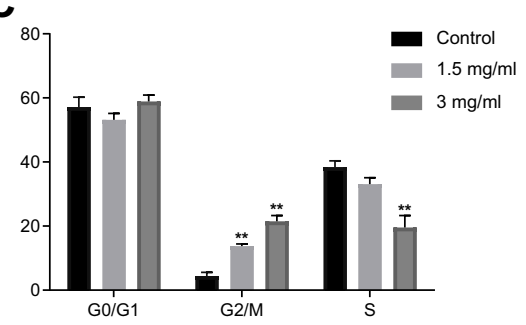

Figure 2 Oxymatrine induces cell cycle arrest in G2/M phase in SW962 and A43I cells. (A-C) Cycle distribution of SW962 and A43I cells after treatment with different dosages of oxymatrine for $48 \mathrm{~h}$. (D-G) Expression of P2I, cyclin BI and CDC2 in SW962 and A43I cells as detected by Western blotting. GAPDH was used to unify the cycle-related protein expression. ${ }^{*} \mathrm{P}<0.05$, ${ }^{*} \mathrm{P}<0.01$ compared with $0 \mathrm{mg} / \mathrm{mL}$ oxymatrine (control group). 


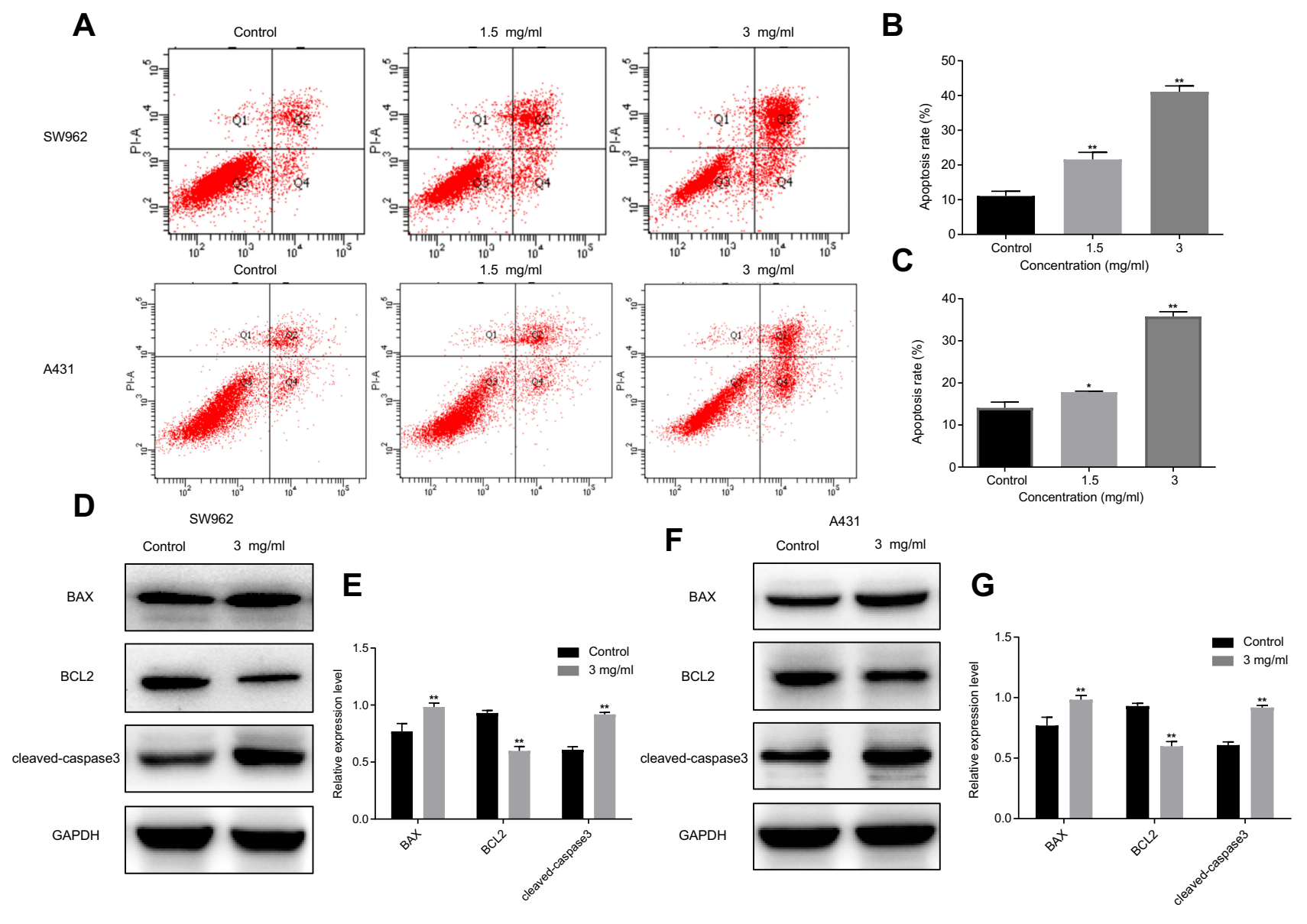

Figure 3 Oxymatrine promotes apoptosis of SW962 and A43I cells. (A-C) Apoptosis rates of SW962 and A43I cells under the action of oxymatrine for $48 \mathrm{~h}$. (D-G) Expression of cleaved-caspase 3, BAX, and BCL2 in SW962 and A43I cells as detected by Western blotting. GAPDH was used to unify the apoptosis-related protein expression. *P $<0.05$, **P $<0.0$ l compared with $0 \mathrm{mg} / \mathrm{mL}$ oxymatrine (control group).

SW962 and A431 cells $(\mathrm{P}<0.05)$. These results showed that oxymatrine promotes apoptosis of SW962 and A431 cells.

\section{Oxymatrine Suppresses Migration and Invasion of SW962 and A43I Cells}

After treatment with different doses of oxymatrine for 48 $\mathrm{h}$, the number of invading cells from $303.0 \pm 12.4 \%$ decreased to $171.3 \pm 13.6 \%$ and $129.6 \pm 10.7 \%$ in SW962 cells, and from $441.0 \pm 8.5 \%$ decreased to $227.3 \pm 18.2 \%$ and $56.7 \pm 13.3 \%$ in A431 cells, respectively. The number of migrating cells from $360.0 \pm 11.1 \%$ decreased to 188.3 $\pm 12.7 \%$ and $97.3 \pm 11.6 \%$ in SW962 cells, and from 549.7 $\pm 17.6 \%$ decreased to $263.3 \pm 22.3 \%$ and $120.7 \pm 11.2 \%$ in A431 cells, respectively $(\mathrm{P}<0.05$; Figure $4 \mathrm{~A}-\mathrm{F})$. The wound width gradually increased with different concentrations of oxymatrine over 48 h. $(\mathrm{P}<0.05$; Figure $4 \mathrm{G}-\mathrm{J})$. Western blot analysis demonstrated that oxymatrine reduced the expression of MMP9 and MMP2 $(\mathrm{P}<0.05$; Figure $4 \mathrm{~K}-\mathrm{N})$. These outcomes showed that oxymatrine could suppress VSCC cells invasion and migration.

\section{Oxymatrine Inhibits the RAS/RAF/MEK/ ERK Signaling Pathway in SW962 and A43I Cells}

The influence of oxymatrine on the RAS/RAF/MEK/ERK pathway was detected using Western blotting. Oxymatrine markedly attenuated expression levels of RAS, p-RAF, p-MEK, p-ERK, C-MYC, and MMP2, whereas the total protein expression of RAF, MEK, and ERK remained unchanged in both SW962 and A431 cells. Similar results were obtained using salirasib. Also known as S-farnesylthiosalicylic acid (FTS), salirasib is an S-farnesylcysteine analogue that is structurally similar to S-farnesylcysteine symbionts of the RAS protein. Salirasib disrupts the connection between the RAS protein and the plasma membrane by competitively inhibiting 
A

SW962

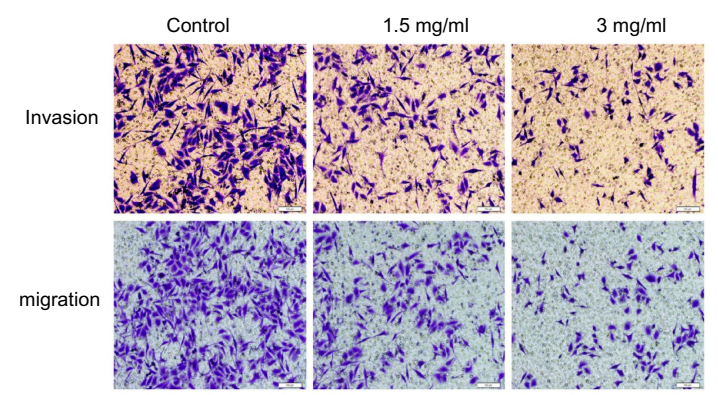

C

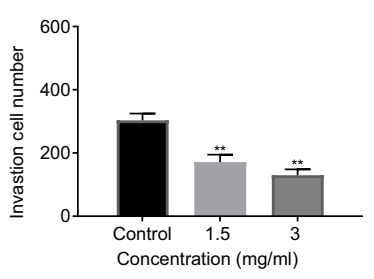

D

G

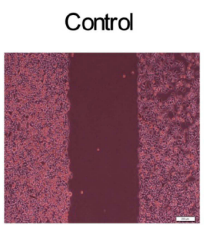

$48 \mathrm{~h}$
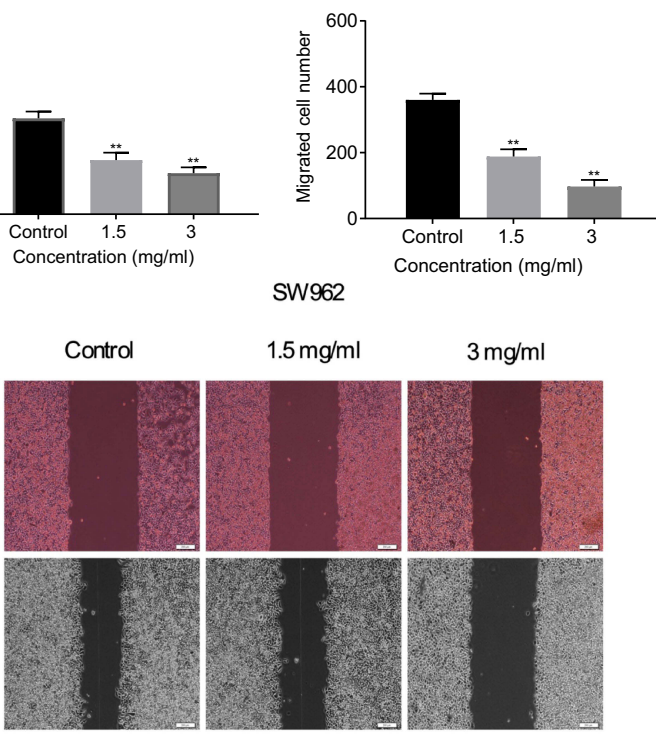

SW962

$1.5 \mathrm{mg} / \mathrm{ml}$
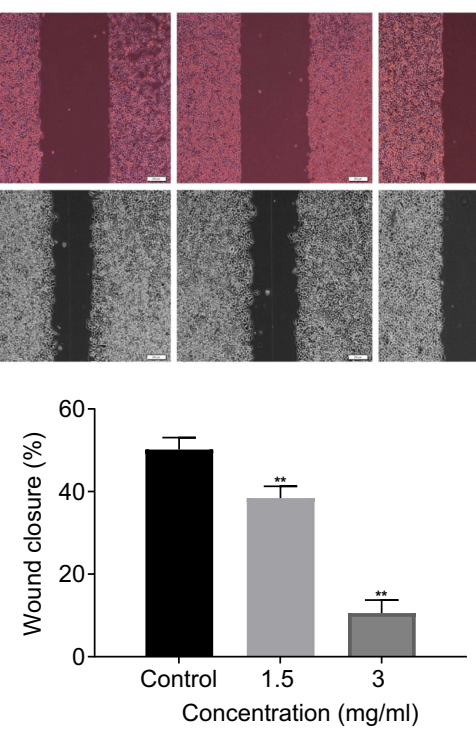

SW962

K
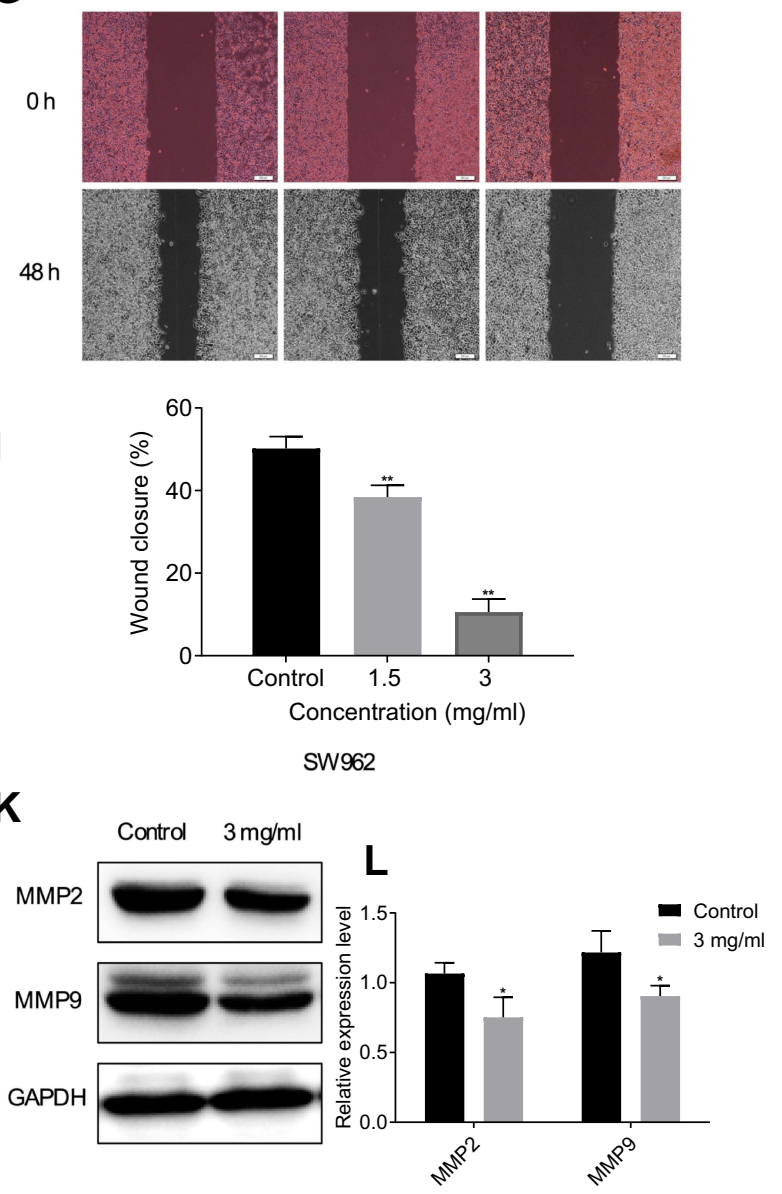

B

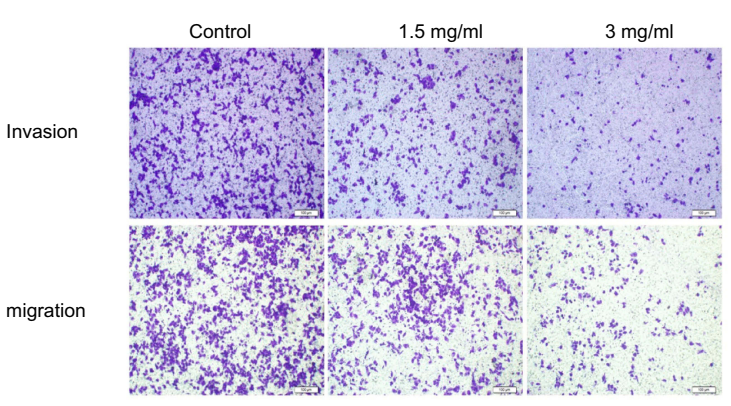

E

F

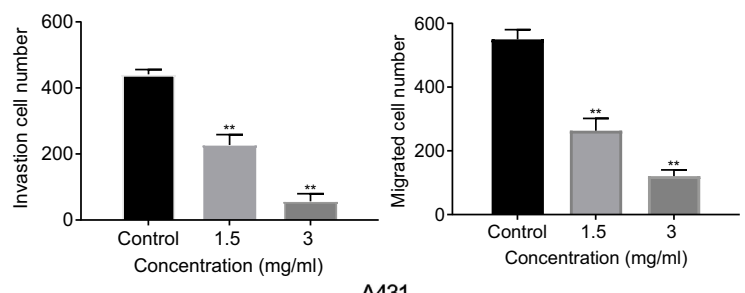

H

Control

$1.5 \mathrm{mg} / \mathrm{ml}$

$3 \mathrm{mg} / \mathrm{ml}$

oh
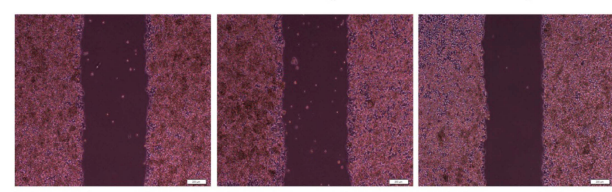

$48 \mathrm{~h}$
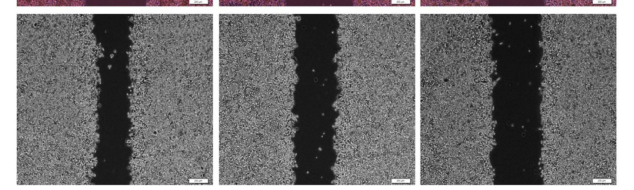

J

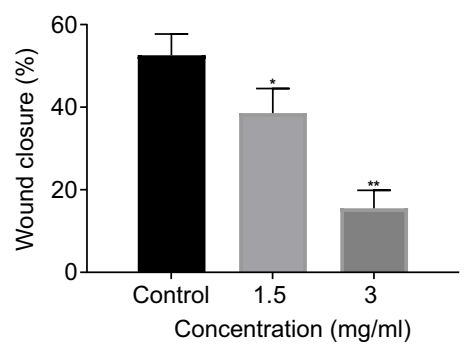

A431

M

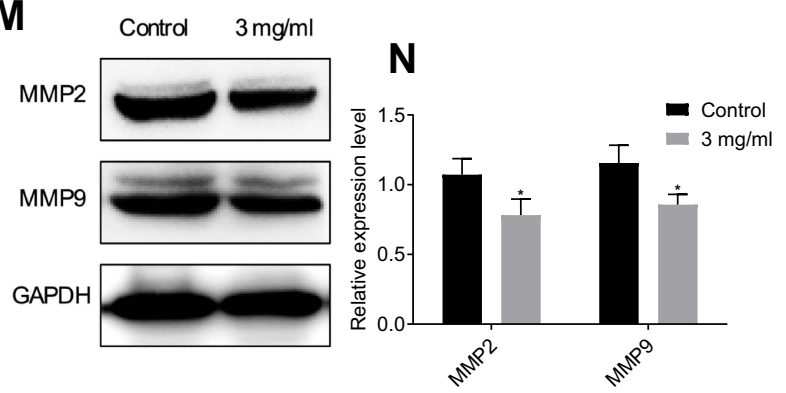

Figure 4 Oxymatrine suppresses migration and invasion of SW962 and A43I cells. (A-F) Under the action of oxymatrine for $48 \mathrm{~h}$, the numbers of migrating and invading SW962 and A43I cells were observed by transwell assay. (G-J) Under the action of Oxymatrine for 48 h, the wound width was examined by wound-healing assay. (K-N) Levels of MMP9 and MMP2 in SW962 and A43I cells as detected by Western blotting. GAPDH was used to unify the migration and invasion-related protein expression. $* \mathrm{P}<0.05, * * \mathrm{P}<0.01$ compared with $0 \mathrm{mg} / \mathrm{mL}$ oxymatrine (control group). 
attachment to the membrane. ${ }^{17,18}$ Studies have shown that salirasib can inhibit RAS-induced signaling pathways, including RAF/MEK/ERK, PI3K/AKT, and RalGEF/Ral, and suppress tumor growth and progression ${ }^{17}(\mathrm{P}<0.05$; Figure 5A-D). Thus, we inferred that oxymatrine could inhibit the RAS/RAF/MEK/ERK pathway in SW962 and A431 cells.

\section{Oxymatrine Reverses TGF- $\beta$ I-Activated RAS/RAF/MEK/ERK Pathway in SW962 and A43I Cells}

To further verify that oxymatrine exerted its antitumor effect via attenuation of the RAS/RAF/MEK/ERK pathway, we treated cells with TGF- $\beta 1$, which is an activator of the RAS signaling pathway. TGF- $\beta 1$ markedly increased the expression of RAS, p-RAF, p-MEK, p-ERK, C-MYC, and MMP2 in SW962 and A431 cells, which indicated that TGF- $\beta 1$ could activate the RAS/RAF/MEK/ERK pathway in these cells. Subsequent oxymatrine and salirasib treatment reversed the effects of TGF- $\beta 1$ in both cell types $(\mathrm{P}<0.05$; Figure 6A-D). Therefore, we concluded that oxymatrine restrains proliferation and migration of SW962 and A431 cells via attenuation of the TGF- $\beta 1$-mediated RAS/RAF/ MEK/ERK signaling pathway.

\section{Discussion}

In most cancers, multiple genes and pathways are often abnormally activated or maladjusted, which tends to promote excessive proliferation and survival of cancer cells. The RAS/RAF/MEK/ERK signaling pathway is involved in the carcinogenesis and metastasis of multiple tumor types. It has been reported that the expression levels of RAS, RAF, MEK, and ERK are associated with lymph node metastasis and overall survival in breast cancer and hepatocellular carcinoma. ${ }^{19,20}$ RAS mutations can impair GTPase hydrolase activity and maintain proteins in an activated state, activating the RAF/MEK/ERK signaling cascade. ${ }^{15}$ In our study, we showed that oxymatrine could inhibit the proliferation and metastasis of VSCC cells in vitro by blocking the RAS/RAF/MEK/ERK pathway.

First, we selected vulvar squamous cell lines SW962 and A431 to study the effects of oxymatrine on cell proliferation. The results showed that oxymatrine suppressed the growth of SW962 and A431 cells in a time- and dose-dependent manner and induced cell cycle arrest in the G2/M phase by downregulating cyclin $\mathrm{B} 1$ and $\mathrm{CDC} 2$ and upregulating $\mathrm{P} 21$ expression. P21 is an inhibitor of cyclin/cyclin-dependent kinase complexes, and cyclin $\mathrm{B} 1$ and $\mathrm{CDC} 2$ are representative mitotic promotors of the G2/M phase. Enhanced cyclin B1/ CDC2 complex activity can accelerate G2/M phase transformation and promote cell mitosis. ${ }^{21} \mathrm{P} 21$ terminates the cell cycle by inhibiting the activity of the cyclin B1/CDC2 complex. $^{22}$ A similar study found that oxymatrine arrested leukemia cells in $\mathrm{G} 2 / \mathrm{M}$ phase. ${ }^{23}$

Second, oxymatrine increased the apoptosis rate of VSCC cells by increasing levels of BAX and cleavedcaspase 3 and decreasing BCL2 expression. BAX and BCL2 are regulators of the mitochondria-dependent intrinsic apoptotic signaling pathway. An increased BAX/BCL2 ratio and decreased mitochondrial membrane potential promote release of cytochrome $\mathrm{c}$ into the cytoplasm to form an apoptotic complex with APAF-1/caspase 9; this cleaves and activates caspase 9 and further activates the downstream effector caspase 3 to initiate apoptosis. ${ }^{24}$ Similar findings in MCF-7 and MDA-MB-231 breast cancer cells show that oxymatrine induces apoptosis by upregulating the BAX/BCL2 ratio and caspase activities. ${ }^{25}$

Third, MMPs degrade most constituents of the extracellular matrix directly and are involved in cancer metastasis. ${ }^{26}$ MMPs are not only important hydrolytic enzymes for extracellular matrix degradation but also are essential proteases in epithelial-mesenchymal transition. In previous reports, MMP2 was observed to be highly expressed in vulvar cancer and promoted cancer cell metastasis through separation of the intercellular matrix. ${ }^{27}$ In the current study, oxymatrine gradually reduced the number of cells involved in invasion and migration, in the case of both SW962 and A431 cells, by reducing expression levels of MMP2 and MMP9. It has been reported that oxymatrine can also downregulate the expression of MMP2 and MMP9 in gallbladder cancer and gastric cancer. $^{10,28}$

The mitogen-activated protein kinase (MAPK) signaling pathway has a focal role in the downstream pathways of RAS; it sequentially consists of RAS, RAF, MEK, and ERK. ${ }^{29}$ Activated RAS first recruits RAF to the cell membrane and promotes its activation, which causes the activation of downstream MEK and ERK through a cascade reaction. Translocation of activated ERK to the cell nucleus directly activates its downstream substrates (of which there are more than 70), leading to changes in gene expression and regulation of various transcription factors. $^{30}$ Continuously activated ERK can enhance the stability of the C-MYC protein and its accumulation in the nucleus by phosphorylating serine at position 62 of the C-MYC protein, which promotes proliferation. ${ }^{31}$ The $\mathrm{MEK} / \mathrm{ERK} / \mathrm{C}-\mathrm{MYC}$ axis has been reported to be involved 


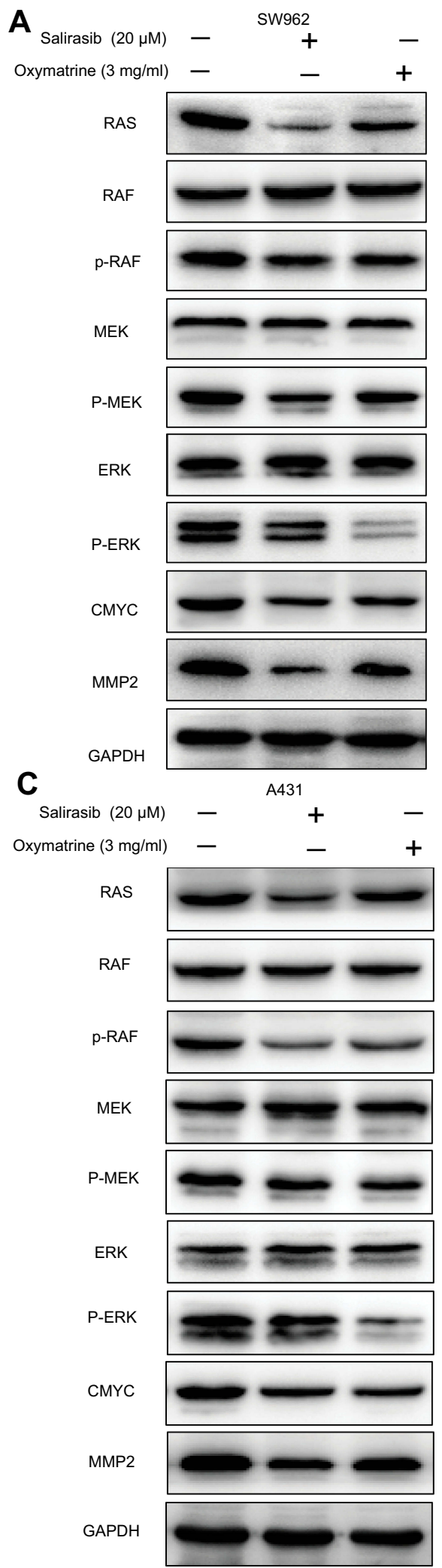

B

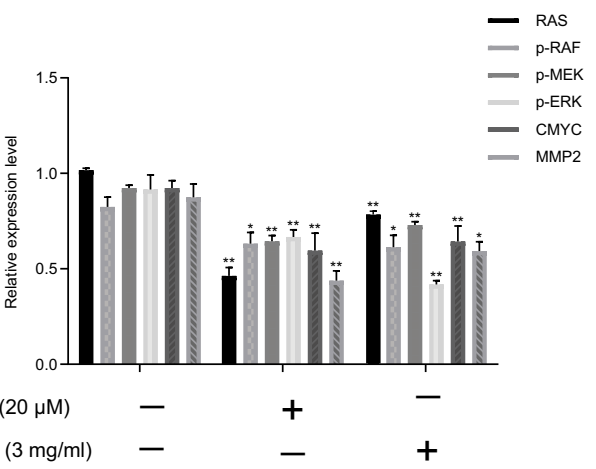

D

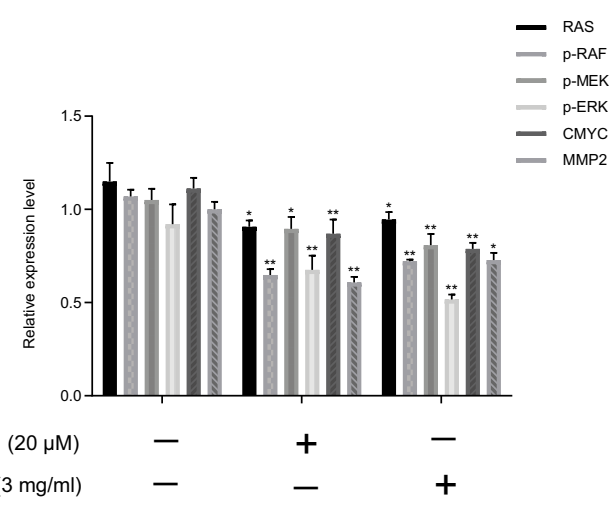

Figure 5 Oxymatrine inhibits RAS/RAF/MEK/ERK signaling pathway in SW962 and A43I cells. SW962 and A43I cells were treated with or without oxymatrine (3 mg/mL) and salirasib $(20 \mu \mathrm{M})$ for $48 \mathrm{~h}$. (A-D) Protein expression of RAS, RAF, MEK, ERK, p-RAF, p-MEK, p-ERK, C-MYC, and MMP2 as detected by Western blotting. *P<0.05, $* * \mathrm{P}<0.01$ compared with $0 \mathrm{mg} / \mathrm{mL}$ oxymatrine (control group). 
A

\begin{tabular}{cccccc} 
A & & \multicolumn{3}{c}{ SW962 } \\
TGF- $\beta 1$ & $(10 \mathrm{ng} / \mathrm{ml})$ & - & + & + & + \\
Salirasib & $(20 \mu \mathrm{M})$ & - & - & + & - \\
Oxymatrine & $(3 \mathrm{mg} / \mathrm{ml})$ & - & - & - & +
\end{tabular}

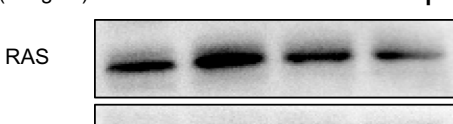

RAF

P-RAF

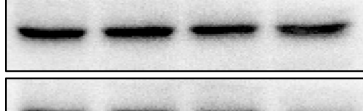

P-RAF

MEK

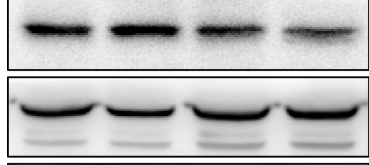

P-MEK

ERK

P-ERK
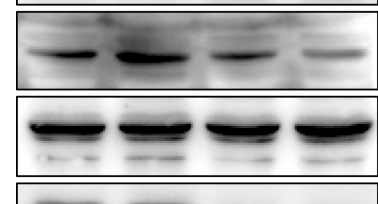

P-ERK

CMYC

MMP2

GAPDH
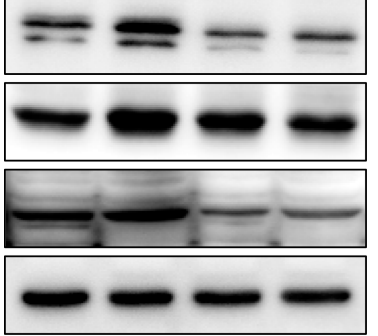

C
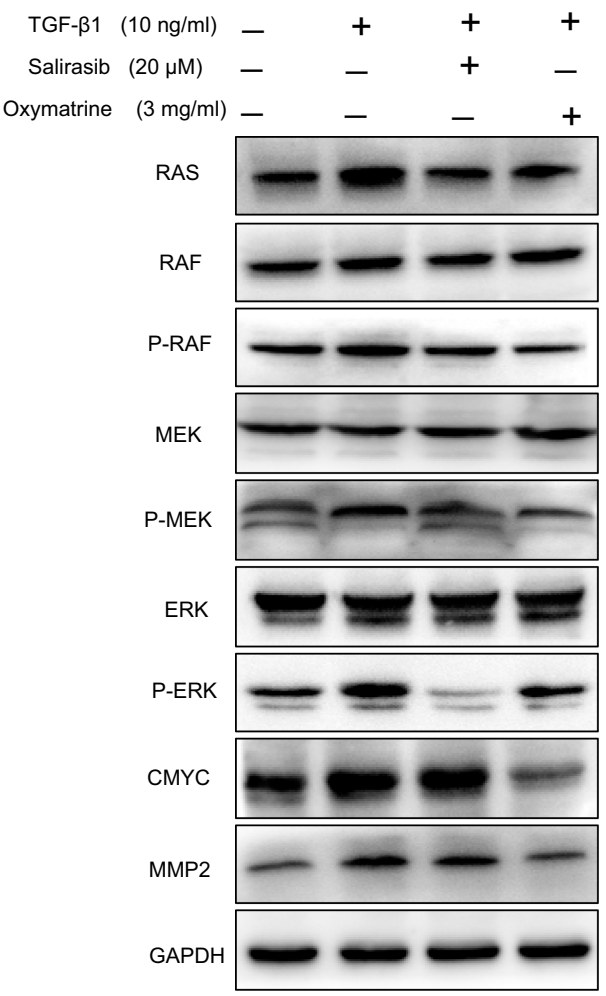

B

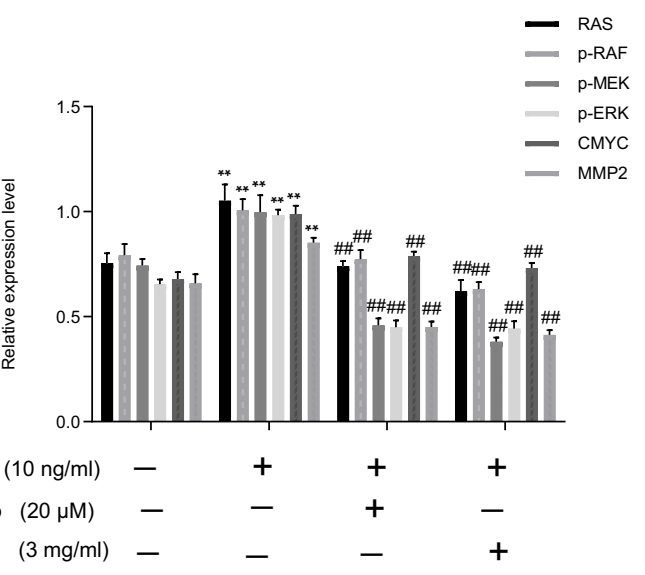

D

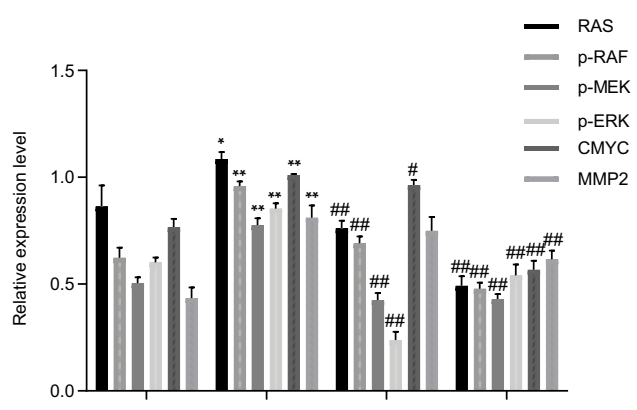

TGF- $\beta 1(10 \mathrm{ng} / \mathrm{ml}) \quad-\quad+\quad+\quad+$

Salirasib $(20 \mu \mathrm{M}) \quad-\quad-\quad+$

Oxymatrine $(3 \mathrm{mg} / \mathrm{ml}) \quad-\quad-\quad-\quad-\quad+$

Figure 6 Oxymatrine reverses TGF- $\beta$ I-mediated RAS/RAF/MEK/ERK pathway in SW962 and A43I cells. Cells were pretreated with TGF- $\beta$ I for I 2 h, and then stimulated with oxymatrine and salirasib for $48 \mathrm{~h}$. (A-D) Protein expression of RAS, RAF, MEK, ERK, p-RAF, p-MEK, p-ERK, C-MYC, and MMP2 investigated by Western blotting. ${ }^{*} \mathrm{P}<0.05$, ${ }^{*} * \mathrm{P}<0.01$ compared with TGF- $\beta \mathrm{I}(-) /$ oxymatrine(-)/salirasib(-) group, ${ }^{\#} \mathrm{P}<0.05,{ }^{\#} \mathrm{P}<0.0 \mathrm{I}$ compared with TGF- $\beta \mathrm{I}(+) / 0 x y m a t r i n e(-) /$ salirasib(-) group. 
in regulation of prostate cancer cell growth. ${ }^{32}$ Studies have shown that increasing phosphorylation levels in the RAS/ $\mathrm{RAF} / \mathrm{MEK} / \mathrm{ERK}$ pathway increases the expression of oncogene C-MYC in breast cancer cells. ${ }^{33}$ Our results support the regulation of C-MYC expression by the RAS/RAF/MEK/ERK pathway. The promoter sequence of MMP2 contains binding sites for two transcription factors, AP-1 and ETS. ${ }^{34}$ The RAS/RAF/MEK/ERK signaling pathway has been shown to be an important pathway for AP-1 and ETS activation. ${ }^{35}$ ERK may enhance the expression levels of MMP2 by activating the transcription factor AP-1. Previous studies have shown that blocking the MAPK/ERK signaling cascade can inhibit MMP2 expression and inhibit the metastasis of oral squamous cell carcinoma. ${ }^{36}$ Our results showed that oxymatrine not only reduced expression levels of RAS, p-RAF, p-MEK, and p-ERK, but also those of C-MYC and MMP2, similar to the effects of salirasib. Inhibition of upstream protein expression can reduce expression of downstream proteins in the same signaling pathway. Therefore, we speculated that oxymatrine may weaken the RAS/RAF/MEK/ERK signaling pathway and reduce the expression of C-MYC and MMP2, which inhibit the proliferation and invasion of VSCC cells.

In order to further verify the anti-tumor mechanisms of oxymatrine, we used TGF- $\beta 1$ to stimulate SW962 and A431 cells. TGF- $\beta 1$, which is an activator of the RAS signaling pathway, directly phosphorylates ShcA by activating $\mathrm{T} \beta \mathrm{R} 1$ and induces it to form a conjugated complex with Grb2/SOS. ${ }^{37}$ Under the stimulation of ShcA/Grb2/ SOS, GTP replaces GDP and binds to RAS, and RAS is activated. The results showed that TGF- $\beta 1$ increased levels of RAS, p-RAF, p-MEK, p-ERK, C-MYC, and MMP2, whereas oxymatrine and RAS inhibitor salirasib reversed these effects, further confirming that oxymatrine may suppress proliferation and migration by blocking the RAS/ RAF/MEK/ERK pathway in VSCC cells.

\section{Conclusion}

In conclusion, our study provided the first evidence that oxymatrine can inhibit proliferation and migration and induce apoptosis of VSCC cells in vitro. Furthermore, we demonstrated that oxymatrine may exert its therapeutic effects via attenuation of the RAS/RAF/MEK/ERK pathway. These results may lead to a more effective treatment strategy for the treatment of VSCC.

\section{Disclosure}

The authors report no conflicts of interest in this work.

\section{References}

1. Nooij LS, Brand FA, Gaarenstroom KN, Creutzberg CL, de Hullu JA, van Poelgeest MI. Risk factors and treatment for recurrent vulvar squamous cell carcinoma. Crit Rev Oncol Hematol. 2016;106:1-13. doi:10.1016/j.critrevonc.2016.07.007

2. Weinberg D, Gomez-martinez RA. Vulvar cancer. Obstet Gynecol Clin North Am. 2019;46(1):125-135. doi:10.1016/j.ogc.2018.09.008

3. Lu ML, Xiang XH, Xia SH. Potential signaling pathways involved in the clinical application of oxymatrine. Phytother Res. 2016;30 (7): 1104-1112.

4. Chen Y, Chen L, Zhang JY, et al. Oxymatrine reverses epithelial-mesenchymal transition in breast cancer cells by depressing alphabeta3 integrin/FAK/PI3K/Akt signaling activation. Onco Targets Ther. 2019;12:6253-6265.

5. Li W, Yu X, Tan S, Liu W, Zhou L, Liu H. Oxymatrine inhibits non-small cell lung cancer via suppression of EGFR signaling pathway. Cancer Med. 2018;7(1):208-218.

6. Pei Z, Zeng J, Gao Y, et al. Oxymatrine inhibits the proliferation of CaSki cells via downregulating HPV16E7 expression. Oncol Rep. 2016;36(1):291-298.

7. Li S, Zhang Y, Liu Q, et al. Oxymatrine inhibits proliferation of human bladder cancer T24 cells by inducing apoptosis and cell cycle arrest. Oncol Lett. 2017;13(6):4453-4458.

8. Chen K, Zhu P, Ye J, et al. Oxymatrine inhibits the migration and invasion of hepatocellular carcinoma cells by reducing the activity of MMP-2/-9 via regulating p38 signaling pathway. J Cancer. 2019;10 (22):5397-5403.

9. Dai Z, Wang L, Wang X, et al. Oxymatrine induces cell cycle arrest and apoptosis and suppresses the invasion of human glioblastoma cells through the EGFR/PI3K/Akt/mTOR signaling pathway and STAT3. Oncol Rep. 2018;40:867-876.

10. Guo B, Zhang T, Su J, Wang K, Li X. Oxymatrine targets EGFR (p-Tyr845) and inhibits EGFR-related signaling pathways to suppress the proliferation and invasion of gastric cancer cells. Cancer Chemother Pharmacol. 2015;75(2):353-363. doi:10.1007/s00280014-2651-1

11. Samatar AA, Poulikakos PI. Targeting RAS-ERK signalling in cancer: promises and challenges. Nat Rev Drug Discov. 2014;13 (12):928-942. doi:10.1038/nrd4281

12. Trietsch MD, Spaans VM, Ter Haar NT, et al. CDKN2A(p16) and HRAS are frequently mutated in vulvar squamous cell carcinoma. Gynecol Oncol. 2014;135(1):149-155. doi:10.1016/j.ygyno.2014.07.094

13. Burotto M, Chiou VL, Lee JM, Kohn EC. The MAPK pathway across different malignancies: a new perspective. Cancer. 2014;120 (22):3446-3456. doi:10.1002/cncr.28864

14. Palisoul ML, Mullen MM, Feldman R, Thaker PH. Identification of molecular targets in vulvar cancers. Gynecol Oncol. 2017;146 (2):305-313. doi:10.1016/j.ygyno.2017.05.011

15. Peyssonnaux C, Eychène A. The Raf/MEK/ERK pathway: new concepts of activation. Biol Cell. 2001;93(1-2):53-62. doi:10.1016/ S0248-4900(01)01125-X

16. Omholt K, Grafstrom E, Kanter-lewensohn L, Hansson J, Ragnarsson-olding BK. KIT pathway alterations in mucosal melanomas of the vulva and other sites. Clin Cancer Res. 2011;17 (12):3933-3942. doi:10.1158/1078-0432.CCR-10-2917

17. Rotblat B, Ehrlich M, Haklai R, Kloog Y. The Ras inhibitor farnesylthiosalicylic acid (salirasib) disrupts the spatiotemporal localization of active Ras: a potential treatment for cancer. Method Enzymol. 2008;439(21):467-489. 
18. Sugita S, Enokida H, Yoshino H, et al. HRAS as a potential therapeutic target of salirasib RAS inhibitor in bladder cancer. Int J Oncol. 2018;53(2):725-736. doi:10.3892/ijo.2018.4435

19. Chen L, Shi Y, Jiang CY, Wei LX, Wang YL, Dai GH. Expression and prognostic role of pan-Ras, Raf-1, pMEK1 and pERK1/2 in patients with hepatocellular carcinoma. Eur J Surg Oncol. 2011;37 (6):513-520. doi:10.1016/j.ejso.2011.01.023

20. Shao GL, Wang MC, Fan XL, et al. Correlation between Raf/MEK/ ERK signaling pathway and clinicopathological features and prognosis for patients with breast cancer having axillary lymph node metastasis. Technol Cancer Res Treat. 2018;17:1-11. doi:10.1177/ 1533034617754024

21. Song L, Han X, Jia C, et al. Porcine reproductive and respiratory syndrome virus inhibits MARC-145 proliferation via inducing apoptosis and $\mathrm{G} 2 / \mathrm{M}$ arrest by activation of $\mathrm{Chk} / \mathrm{Cdc} 25 \mathrm{C}$ and $\mathrm{p} 53 / \mathrm{p} 21$ pathway. Virol J. 2018;15(1):169. doi:10.1186/s12985-018-1081-9

22. Touny LH, Banerjee PP. Identification of both Myt-1 and Wee-1 as necessary mediators of the p21-independent inactivation of the cdc-2/ cyclin B1 complex and growth inhibition of TRAMP cancer cells by genistein. Prostate. 2006;66(14):1542-1555. doi:10.1002/pros.20495

23. Liu J, Yao Y, Ding H, Chen R. Oxymatrine triggers apoptosis by regulating Bcl-2 family proteins and activating caspase-3/caspase- 9 pathway in human leukemia HL-60 cells. Tumour Biol. 2014;35 (6):5409-5415. doi:10.1007/s13277-014-1705-7

24. Birkinshaw RW, Czabotar PE. The BCL-2 family of proteins and mitochondrial outer membrane permeabilisation. Semin Cell Dev Biol. 2017;72:152-162. doi:10.1016/j.semcdb.2017.04.001

25. Wu J, Cai Y, Li M, et al. Oxymatrine promotes S-phase Arrest and inhibits cell proliferation of human breast cancer cells in vitro through mitochondria-mediated apoptosis. Biol. Pharm. Bull. 2017;40(8):1232-1239. doi:10.1248/bpb.b17-00010

26. Bjorklund M, Koivunen E. Gelatinase-mediated migration and invasion of cancer cells. Biochim Biophys Acta. 2005;1755(1):37-69. doi:10.1016/j.bbcan.2005.03.001
27. Bovo AC, da Silva ID, Takita LC, et al. A comparative study of MMP-2 in vulvar neoplasms. Gynecol Oncol. 2004;93(2):454-457. doi:10.1016/j.ygyno.2004.01.049

28. Qian L, Li X, Ye P, et al. Oxymatrine induces apoptosis and inhibits invasion in gallbladder carcinoma via PTEN/PI3K/AKT pathway. Cytotechnology. 2018;70(1):83-94. doi:10.1007/s10616-017-0153-9

29. Yang S, Liu G. Targeting the Ras/Raf/MEK/ERK pathway in hepatocellular carcinoma. Oncol Lett. 2017;13(3):1041-1047.

30. Steelman LS, Franklin RA, Abrams SL, et al. Roles of the Ras/Raf/ MEK/ERK pathway in leukemia therapy. Leukemia. 2011;25 (7):1080-1094.

31. Sears R, Nuckolls F, Haura E, et al. Multiple Ras-dependent phosphorylation pathways regulate Myc protein stability. Genes Dev. 2000; 14:2501-2514.

32. Chu J, Li Y, Deng Z, et al. IGHG1 regulates prostate cancer growth via the MEK/ERK/c-Myc pathway. Biomed Res Int. 2019;2019:1-10.

33. Paliouras M, Diamandis EP. Intracellular signaling pathways regulate hormone-dependent kallikrein gene expression. Tumour Biol. 2008;29(2):63-75.

34. Kajanne R, Miettinen P, Mehlem A, et al. EGF-R regulates MMP function in fibroblasts through MAPK and AP-1 pathways. $J$ Cell Physiol. 2007;212(2):489-497.

35. Hollenhorst PC. RAS/ERK pathway transcriptional regulation through ETS/AP-1 binding sites. Small GTPases. 2012;3(3):154-158.

36. Fang C-Y, Wu C-Z, Chen P-N, et al. Antimetastatic potentials of salvianolic acid $\mathrm{A}$ on oral squamous cell carcinoma by targeting MMP-2 and the c-Raf/MEK/ERK pathway. Environ Toxicol. 2018;33(5):545-554

37. Lee MK, Pardoux $\mathrm{C}$, Hall MC, et al. TGF- $\beta$ activates Erk MAP kinase signalling through direct phosphorylation of ShcA. EMBO J. 2007;26:3957-3967.

\section{Publish your work in this journal}

Cancer Management and Research is an international, peer-reviewed open access journal focusing on cancer research and the optimal use of preventative and integrated treatment interventions to achieve improved outcomes, enhanced survival and quality of life for the cancer patient.
The manuscript management system is completely online and includes a very quick and fair peer-review system, which is all easy to use. Visit http://www.dovepress.com/testimonials.php to read real quotes from published authors. 